\title{
Ostwald ripening with size-dependent rates: Similarity and power-law solutions
}

\author{
Giridhar Madras ${ }^{a)}$ \\ Department of Chemical Engineering, Indian Institute of Science, Bangalore 560 012, India \\ Benjamin J. McCoy \\ Department of Chemical Engineering and Materials Science, University of California, Davis, \\ California 95616
}

\begin{abstract}
The dynamics of Ostwald ripening is treated by cluster distribution kinetics represented by a population balance equation that also describes growth or dissolution. Unlike simple crystal growth driven by supersaturation, the smaller, more soluble clusters in the distribution dissolve during ripening near equilibrium and vanish when they reach the critical nucleus size. Larger clusters accordingly grow as the supersaturation decreases. The long-time asymptotic result of the numerical solution of the scaled population balance equation is power-law decrease of cluster number and growth of average cluster mass, $C^{\text {avg }}(\theta)$. The cluster distribution approximates an exponential self-similar solution, and eventually narrows until but one large cluster remains, satisfying the mass balance. A previous theory is here extended to include mass-dependent rate coefficients for growth and dissolution that satisfy microscopic reversibility. The asymptotic power-law growth, $C^{\text {avg }}$ $\sim \theta^{1 /(4 / 3-\lambda)}$, is determined by the power $\lambda$ on the mass for rate coefficients. The power is $\lambda=1 / 3$ for diffusion-controlled and $\lambda=2 / 3$ for surface-controlled processes. Experimentally observed ripening behavior is realized by an apt choice of $\lambda$ for a given time range.
\end{abstract}

\section{INTRODUCTION}

The dynamics of cluster growth and dissolution finds application in many scientific fields, including biology, ${ }^{1}$ aerosol science, ${ }^{2}$ materials science, ${ }^{3}$ surface science, ${ }^{4}$ geology, ${ }^{5}$ and chemical engineering. ${ }^{6}$ The growth dynamics for clusters such as crystals, particles, or droplets in solidliquid, solid-vapor, liquid-vapor, liquid-liquid, or solidsolid phase transitions can be understood by examining the time evolution of cluster size distributions (CSDs). The kinetics, thermodynamics, and transport processes for the system influence the evolution. Modeling the temporal evolution can be accomplished by population balance equations (PBEs) that describe the distribution dynamics by mass balances expressed as integro-differential equations for the CSD. $^{7-9}$ At the molecular level monomers reversibly attach and dissociate at the cluster surface, causing dispersive growth similar to polymerization, and leading eventually to a dynamic equilibrium. Our overall aim is to develop a general and comprehensive approach to such particulate dynamics by means of distribution kinetics expressed through PBEs.

In condensed-matter physics, Ostwald ripening, or coarsening, is the final stage of a first-order phase transition for condensation of a metastable phase. ${ }^{10}$ The first stage is nucleation, and the second stage is dispersed cluster growth by reversible monomer deposition. In the final stage, smaller clusters dissolve and give up their monomers to larger clusters. The explanation for such ripening is based on the

\footnotetext{
a) Author to whom all correspondence should be addressed; electronic mail: giridhar@chemeng.iisc.ernet.in
}

Gibbs-Thomson equation, which relates the ratio of interfacial energy to thermal energy such that smaller clusters are more soluble than larger ones. Clusters become less stable as they become smaller, and eventually dissolve, transferring their mass to the solution and on to larger clusters. Clusters smaller than the critical nucleus are here considered completely unstable, and hence they instantaneously disappear. ${ }^{5,11}$ The ultimate state is reached when only one cluster remains in equilibrium with the monomer solution. ${ }^{2}$ The eventual evolution to a single cluster is slow and follows a power-law relation. ${ }^{10,12}$ For example, in the crystallization of biological macromolecules from precipitates, ${ }^{2}$ Ostwald ripening occurs over a period of months. Time scales on the order of millions of years may be required for geological ripening phenomena. ${ }^{5}$

In our population balance equation formulation, a rate coefficient describes the rate with which collisions between a monomer and a cluster result in attachment. The size dependency of this growth rate coefficient affects the shape of the resultant cluster size distribution. The growth of clusters may also be influenced by diffusion and convection effects. As with nucleation, solubility affects growth rates, which depend on the rate at which molecules reach the growing cluster surface. For protein crystallization, Feher and $\mathrm{Kam}^{13}$ have shown that the regions surrounding growing crystals have lower protein concentration relative to the surrounding solution. The rate of diffusion of proteins in and out of these regions around the growing crystal limits the growth. Another case is when the surface process controls the growth. Thus, the rate coefficient for crystal growth realistically de- 
pends on size. The dissolution rate is represented as a rate coefficient times the cluster concentration, where surface conditions and mass transfer from the surface can influence the rate. For both diffusion and surface controlled cases, the deposition and dissolution rates increase with cluster size.

Recently, Madras and $\mathrm{McCoy}^{7}$ formulated a new approach to Ostwald ripening that accounted for the evolution of the CSD in terms of spherical cluster mass. This approach is based on distribution kinetics with single-monomer concurrent addition and dissociation. The equations are applicable to the reversible process and can be used to quantitatively model growth, dissolution, or ripening phenomena. The reversibility ensures that a closed system relaxes to dynamic equilibrium with monomer addition and dissociation continuing at equilibrium. This theory also showed the evolution of the CSDs to a single large cluster. However, the ripening theory was restricted to rate coefficients for growth and dissolution independent of size. Coalescence was ignored to focus on growth and dissolution behavior, but aggregation and deaggregation processes can be incorporated via a PBE approach. ${ }^{14}$ Diffusion-controlled growth is restricted to widely separated particles, but it is possible that other growth mechanisms can be represented by treating size dependence as an experimental parameter. Reviews ${ }^{15,16}$ of cluster nucleation and growth by ripening have been published. A recent paper ${ }^{17}$ involves surfactants added to the solution, such that the presence of micelles changes the ripening problem by including a micellar phase in addition to the solution and clusters.

Conventional theories are based on solving an approximate first-order differential equation for cluster growth of the CSD. ${ }^{18-23}$ Most of these theories also approximate the exponential in the Gibbs-Thomson equation by a linear term. Many models do not explicitly represent the evolution of the CSD to a single large cluster, where the variance of the CSD would approach zero (polydispersity index approaches unity). Moreover, some ripening models apply only to a few particles rather than to the distribution of cluster sizes. A key feature of the present theory is that the relationship between interfacial and thermal energy is applied in two ways: to determine the increased solubility of smaller particles relative to large particles, and to represent the critical nucleus size. In classical nucleation theory, clusters smaller than the critical size are unstable, and can exist only as fluctuations. Accordingly, we assume that clusters smaller than the critical size instantaneously vanish.

As described previously, the evolution of CSDs can be influenced by the size-dependent rates for cluster growth and dissolution. The goal of the present work is to extend our earlier treatment ${ }^{7}$ to include rate coefficients that depend on cluster mass, and satisfy microscopic reversibility. The coefficients are determined by diffusion- or surface-related processes. The theory with size-dependent rates demonstrates that ripening continues until cluster mass is accumulated into a single large cluster, and the polydispersity index thus decreases to unity. We show that the final equilibrium state is approached by an asymptotic process independently of initial conditions. By accounting in the rate expressions for diffusion- or reaction-controlled growth and dissolution, the theory provides a framework for understanding the observed features of Ostwald ripening, including self-similar CSDs and power-law increase of average cluster size with time.

After introducing the theoretical model and the governing differential equations in scaled form (Sec. II), we numerically solve for the CSD evolution (Sec. III) over a large time range. The asymptotic long-time power-law solution is derived from the moment equations (Sec. IV). We present a new relationship between the exponent, $\lambda$, for the rate coefficient and the power, $b=(4 / 3-\lambda)^{-1}$. Results are discussed by comparison with other models and with experimental observations. In Sec. V conclusions about the validity of the present theory and of previous theories of ripening are discussed.

\section{THEORETICAL MODEL}

For cluster ripening in a batch system, the CSD is defined by $c(x, t) d x$, representing the concentration of clusters at time $t$ in the differential mass range $(x, x+d x)$. Moments are defined as integrals over the mass,

$$
c^{(n)}(t)=\int_{o}^{\infty} c(x, t) x^{n} d x .
$$

The zeroth moment, $c^{(0)}(t)$, and the first moment, $c^{(1)}(t)$, are the time-dependent molar (or number) concentration of clusters and the cluster mass concentration (mass/volume), respectively. The ratio of the two is the average cluster mass, $c^{\text {avg }}=c^{(1)} / c^{(0)}$. The variance, $c^{\mathrm{var}}=c^{(2)} / c^{(0)}-\left[c^{\mathrm{avg}}\right]^{2}$, and the polydispersity, $c^{\mathrm{pd}}=c^{(2)} c^{(0)} / c^{(1) 2}$, are measures of the CSD broadness. The molar concentration, $m^{(0)}(t)$, of solute monomer of molecular weight $x_{m}$ is the zeroth moment of the monomer distribution, $m(x, t)=m^{(0)}(t) \delta\left(x-x_{m}\right)$.

The deposition or condensation process by which monomers of mass $x^{\prime}=x_{m}$ are reversibly added to or dissociated from a cluster of mass $x$ can be written as the reaction-like process,

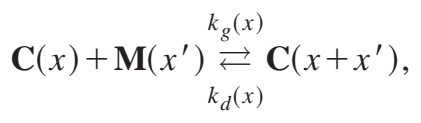

where $\mathbf{C}(x)$ is the cluster of mass $x$ and $\mathbf{M}\left(x^{\prime}=x_{m}\right)$ is the monomer. This process intrinsically conserves mass, and is most naturally represented by balance equations in terms of mass $x$ rather than cluster radius $r$. The balance equations governing the cluster distribution, $c(x, t)$, and the monomer distribution, $m(x, t)$, are thus based on mass conservation: ${ }^{7}$

$$
\begin{aligned}
\partial c(x, t) / \partial t= & -k_{g}(x) c(x, t) \int_{0}^{\infty} m\left(x^{\prime}, t\right) d x^{\prime}+\int_{0}^{x} k_{g}(x \\
& \left.-x^{\prime}\right) c\left(x-x^{\prime}, t\right) m\left(x^{\prime}, t\right) d x^{\prime}-k_{d}(x) c(x, t) \\
& +\int_{x}^{\infty} k_{d}\left(x^{\prime}\right) c\left(x^{\prime}, t\right) \delta\left(x-\left(x^{\prime}-x_{m}\right)\right) d x^{\prime} \\
& -I \delta\left(x-x^{*}\right)
\end{aligned}
$$

and 


$$
\begin{aligned}
\partial m(x, t) / \partial t= & -m(x, t) \int_{0}^{\infty} k_{g}\left(x^{\prime}\right) c\left(x^{\prime}, t\right) d x^{\prime} \\
& +\int_{x}^{\infty} k_{d}\left(x^{\prime}\right) c\left(x^{\prime}, t\right) \delta\left(x-x_{m}\right) d x^{\prime} \\
& +I \delta\left(x-x^{*}\right) x^{*} / x_{m} .
\end{aligned}
$$

According to the molecularity of Eq. (2.2), addition reactions are second order in $c(x, t)$ and $m(x, t)$, whereas dissociation reactions are first order in $c(x, t)$. Nucleation of clusters of mass $x^{*}$ at rate $I$ are source terms or, in this case, sink terms for denucleation, which occurs when clusters shrink to their critical size, $x^{*}$, and then spontaneously vanish. The difference between ordinary dissolution due to concentration driving forces and total disintegration due to thermodynamic instability is thus underscored. For ordinary particle growth or dissolution, we set $I=0$. Initial conditions for Eqs. (2.3) and (2.4) are $c(x, t=0)=c_{0}(x)$, and $m(x, t$ $=0)=m_{0}^{(0)} \delta\left(x-x_{m}\right)$.

We consider ripening to occur when competing growth and dissolution processes for large and small clusters cause (i) large particles to grow while smaller particles shrink, and (ii) denucleation that decreases the number of clusters. The CSD changes according to Eq (2.3), which becomes-when the integrations over the Dirac distributions are performedthe finite-difference differential equation,

$$
\begin{aligned}
\partial c(x, t) / \partial t= & -k_{g}(x) c(x, t) m^{(0)}+k_{g}\left(x-x_{m}\right) c(x \\
& \left.-x_{m}, t\right) m^{(0)}-k_{d}(x) c(x, t)+k_{d}\left(x+x_{m}\right) \\
& \times c\left(x+x_{m}, t\right)-I \delta\left(x-x^{*}\right) .
\end{aligned}
$$

Equation (2.5) shows that $c(x, t)$ increases by addition of mass $x_{m}$ to the reactant of mass $\left(x-x_{m}\right)$ and decreases by the loss of reactant of mass $x$. The dissociation of mass $x_{m}$ from reactant of mass $\left(x+x_{m}\right)$ increases $c(x, t)$ while the loss of reactant of mass $x$ decreases $c(x, t)$. Equation (2.5) resembles an equation of Xia and Zinke-Allmang, ${ }^{24}$ except for the absence of a denucleation term, and can be expanded for $x_{m} \ll x$ to convert the differences into differentials. ${ }^{7,24}$

At equilibrium, $\partial c / \partial t=0$ and Eq. (2.5) becomes

$$
\begin{gathered}
m_{\mathrm{eq}}^{(0)}=-\left[k_{d}\left(x+x_{m}\right) c\left(x+x_{m}\right)-k_{d}(x) c(x)\right] / \\
{\left[k_{g}\left(x-x_{m}\right) c\left(x-x_{m}\right)-k_{g}(x) c(x)\right] .}
\end{gathered}
$$

As stated previously Eq. (2.6) holds for equilibrium, so that nucleation cannot occur $(I=0)$. Expanding the numerator and denominator around $x$ for $x_{m} \ll x$ gives

$$
m_{\mathrm{eq}}^{(0)}=d\left[k_{d}(x) c(x)\right] / d x / d\left[k_{g}(x) c(x)\right] / d x .
$$

We can integrate the differentials between $c(x=0)=0$ and $c_{\text {eq }}(x)$ to get

$$
k_{d}(x)=m_{\mathrm{eq}}^{(0)} k_{g}(x),
$$

which is a statement of microscopic reversibility (detailed balance). ${ }^{25,26}$ Thus, with rate coefficients for a cluster of mass, $x$, if we have an expression for $k_{g}(x)$, we can calculate $k_{d}(x)$.
A monomer that attaches to a cluster must diffuse through the solution to react at the cluster surface. ${ }^{27}$ Such diffusion-controlled reactions have a rate coefficient represented $^{28}$ by

$$
k_{g}=4 \pi\left(D_{c}+D_{m}\right)\left(r_{c}+r_{m}\right)
$$

in terms of diffusion coefficients $(D)$ and radii $(r)$ for spherical cluster and monomer. Because $D_{c} \ll D_{m}$ and $r_{c} \gg r_{m}$, we have

$$
k_{g}=4 \pi D_{m} r_{c}
$$

where $r_{c}=\left(3 x / 4 \pi \rho_{c}\right)^{1 / 3}$. Thus, we can write

$$
k_{g}(x)=\gamma x^{\lambda},
$$

where $\lambda=1 / 3$ and $\gamma=4 \pi D_{m}\left(3 / 4 \pi \rho_{c}\right)^{1 / 3}$. The $1 / 3$ power on $x$ thus represents diffusion-controlled ripening, and coincides with Binder's ${ }^{12}$ expression.

When growth is limited by monomer attachment and dissociation at the cluster surface, the rate coefficient is proportional to the cluster surface area, ${ }^{24} k_{g} \propto r_{c}^{2}$, so that we can write $k_{g}=\gamma x^{2 / 3}$; thus in Eq. (2.11), $\lambda=2 / 3$ for surfacecontrolled ripening. If the deposition is independent of the surface area, then $k_{g}=\gamma x^{0}$. Other expressions for the rate coefficients that are applicable to cluster growth ${ }^{6,20,24,29}$ may be realistic for complex and combined rate processes.

To establish an expression for the dissolution rate coefficient, we use the Gibbs-Thomson equation,

$$
m_{\mathrm{eq}}^{(0)}=m_{\infty}^{(0)} \exp (\Omega)
$$

with

$$
\Omega=2 \sigma v / r_{c} R T,
$$

where $v$ is monomer molar volume, $\sigma$ is interfacial energy, $R$ is the gas constant, and $T$ is temperature. Substitution into Eq. (2.8) yields

$$
k_{d}(x)=\kappa x^{\lambda} \exp \left[\omega\left(x / x_{m}\right)^{-1 / 3}\right],
$$

where $\kappa=\gamma m_{\infty}^{(0)}$ and $\omega=\left(3 x_{m} / 4 \pi \rho_{c}\right)^{-1 / 3} 2 \sigma v / R T$. Substituting these expressions in Eq. (2.5) yields

$$
\begin{aligned}
\partial c(x, t) / \partial t= & -\gamma x^{\lambda} c(x, t) m^{(0)}(t)+\gamma\left(x-x_{m}\right)^{\lambda} \\
& \times c\left(x-x_{m}, t\right) m^{(0)}(t)-\kappa x^{\lambda} \\
& \times \exp \left[\omega\left(x / x_{m}\right)^{-1 / 3}\right] c(x, t)+\kappa\left(x+x_{m}\right)^{\lambda} \\
& \times \exp \left[\omega\left((x+x m) / x_{m}\right)^{-1 / 3}\right] \\
& \times c\left(x+x_{m}, t\right)-I \delta\left(x-x^{*}\right) .
\end{aligned}
$$

For $\lambda=0$, Eq. (2.15) reduces to the governing equation in our previous work. ${ }^{7}$

Using the dimensionless variables,

$$
\begin{aligned}
& \xi=x / x_{m}, \quad \theta=t \gamma m_{\infty}^{(0)} x_{m}^{\lambda}, \quad S=m^{(0)} / m_{\infty}^{(0)}, \\
& C=c x_{m} / m_{\infty}^{(0)}, \\
& C^{(n)}=c^{(n)} / m_{\infty}^{(0)} x_{m}^{n}, \quad J=I / \gamma m_{\infty}^{(0) 2} x_{m}^{\lambda}
\end{aligned}
$$

in Eq. (2.15) and Eq. (2.4) yields the fully dimensionless equations, 


$$
\begin{aligned}
\partial C(\xi, \theta) / \partial \theta= & S(\theta)\left[-\xi^{\lambda} C(\xi, \theta)+(\xi-1)^{\lambda} C(\xi-1, \theta)\right] \\
& -\xi^{\lambda} \exp \left(\omega \xi^{-1 / 3}\right) C(\xi, \theta) \\
& +(\xi+1)^{\lambda} \exp \left(\omega(\xi+1)^{-1 / 3}\right) \\
& \times C(\xi+1, \theta)-J \delta\left(\xi-\xi^{*}\right)
\end{aligned}
$$

and

$$
d S(\theta) / d \theta=\left[-S(\theta)+e^{\Omega a}\right] C^{(\lambda)}+J \xi^{*}
$$

with initial conditions, $S(\theta=0)=S_{0}$ and $C(\xi, \theta=0)$ $=C_{0}(\xi)$. The ratio of interfacial to thermal energy, or Gibbs-Thomson relationship, gives

$$
\Omega(\xi)=\omega / \xi^{1 / 3}, \quad \xi^{*}=(\omega / \ln S)^{3} .
$$

From Eq. (2.1) the scaled moments are

$$
C^{(n)}(\theta)=\int_{0}^{\infty} C(\xi, \theta) \xi^{n} d \xi
$$

In the formulation of Eq. (2.17), the Gibbs-Thomson equation applies to each cluster rather than to the average cluster; thus, $\Omega$ is a function of $\xi$ and larger clusters grow while smaller clusters dissolve. Equation (2.18) is a moment equation, however, and $\Omega_{a}$ is evaluated at the average-sized cluster, $\Omega_{a}=\omega /\left(C^{\text {avg }}\right)^{1 / 3}$, where $C^{\text {avg }}=C^{(1)} / C^{(0)}$. An alternate treatment is to expand $\left[-S(\theta)+e^{\Omega}\right] \approx \Omega$, which is valid for small $\Omega$ and for $S$ near to its equilibrium value, ${ }^{10,18} S=1$. Then Eq. (2.18) would become

$$
d S(\theta) / d \theta=\omega C^{(\lambda-1 / 3)}+J \xi^{*} .
$$

The computational effect of this approximation becomes negligible as the distribution shifts with time to larger particles.

The powers are equal for the growth and dissociation terms in Eq. (2.17) owing to detailed balancingmicroscopic reversibility, Eq. (2.8). Reif ${ }^{25}$ observes that for a system in equilibrium, microscopic reversibility will in general be valid, so that the probability of occurrence of a process must equal the probability of occurrence of the reverse process. Detailed balance is also a consequence of employing reciprocal relations in reaction processes. ${ }^{26}$

\section{NUMERICAL SOLUTION}

For various values of $\lambda$ we solved the differential Eqs. (2.17) - (2.20) by a Runge-Kutta technique with an adaptive time step with $C(\xi, \theta)$ evaluated sequentially at each time step. Denucleation implies that clusters are removed at every time step of the computation when they shrink to the critical cluster size, $\xi^{*}$, such that the CSD is zero when $\xi \leqslant \xi^{*}$. The cluster moments are calculated by integration of the nonzero CSD from $\xi^{*}$ to $\infty$. Because $C(\xi, \theta)$ lies in the semi-infinite domain, it was converted to a bounded range $(0,1)$ by the mapping function, $\xi=\xi^{*}+\left(C^{\text {avg }}-\xi^{*}\right) y /(1-y)$ with $0 \leqslant y$ $\leqslant 1$. This causes $y$ to vary from 0 to 1 when $\xi$ varies from $\xi^{*}$ to $\infty$. By choosing this mapping, we ensure that when $y$ is centered at 0.5 , the distribution is centered around $C^{\text {avg }}(\theta)$ and is bounded at the lower end by $\xi^{*}$, which increases as $S$ decreases, according to Eq. (2.19). Choosing this grid provides that the mapping is fine in the range of prevalent sizes and coarse at very high and very low sizes. It is, therefore, possible to consider a narrow CSD with a few hundred intervals to do the numerical analysis. The mass variable $(y)$ was divided into 1000 intervals and the adaptive time $(\theta)$ step varied from 0.001 to 0.1 ensuring stability and accuracy at all values of the parameters. $I$ (or $J$ ) is the number of clusters removed at every time step of the computation when they reach the critical cluster size, $x^{*}$. At every time step, the mass balance,

$$
S_{0}+C_{0}^{(1)}=S(\theta)+C^{(1)}(\theta),
$$

was verified.

For generality, we consider an initial gamma distribution with smallest cluster mass, $\xi_{0}^{*}$,

$$
\begin{aligned}
C_{0}(\xi)= & {\left[C_{0}^{(0)} / \Gamma(\alpha) \beta_{0}\right]\left[\left(\xi-\xi^{*}\right) / \beta_{0}\right]^{\alpha-1} } \\
& \times \exp \left[-\left(\xi-\xi_{0}^{*}\right) / \beta_{0}\right],
\end{aligned}
$$

which has the moments

$$
C_{0}^{(n)}=C_{0}^{(0)} \sum_{j=}^{n}\left(\begin{array}{l}
n \\
j
\end{array}\right) \xi_{0}^{*^{n-j}} \beta_{0}^{j} \Gamma(j+\alpha) / \Gamma(\alpha) .
$$

Thus, $C_{0}^{\mathrm{avg}}=\alpha \beta_{0}+\xi_{0}^{*}$ and $C_{0}^{\mathrm{var}}=\alpha \beta_{0} \cdot{ }^{2}$ We choose $\alpha=1$ for an initial exponential distribution, the dimensionless zeroth moment $C_{0}^{(0)}=1$, and the supersaturation $S_{0}=5$. The parameter values, $\omega=2$ and 5, were used in computing the factor in the Gibbs-Thomson equation, $\Omega$, and the critical cluster size, $\xi^{*}$. The initial critical size, $\xi_{0}^{*}$, is 30 when $\omega=S_{0}=5$, and 1.9 when $\omega=2$ and $S_{0}=5$. The initial average cluster mass, $C_{0}^{\text {avg }}$, of course must be larger than $\xi_{0}^{*}$.

Figure 1 shows the evolution of the CSD for $C_{0}^{\mathrm{avg}}=75$ and $\lambda=1 / 3$. The distribution evolves [Fig. 1(a)] from an initial exponential distribution cut off at a value of $\xi=30$, which is the initial critical cluster size, $\xi_{0}^{*}$. As the number of clusters declines and the clusters become larger, the CSD area and height decrease. The critical nucleus mass, represented by the scaled quantity, $\xi^{*}$, increases with time. Clusters smaller than $\xi^{*}$ are totally unstable, and are considered to vanish instantaneously. This is a departure from other theories ${ }^{7,12}$ that assume clusters of all sizes are present in the distribution, with the condition that $\lim _{\xi \rightarrow 0} C(\xi, \theta)=0$. The numerical calculation for ripening ends when the remaining one cluster is in equilibrium with the monomer phase, consistent with the mass balance based on initial total amount of monomer, is thus $C_{\infty}^{(1)}=C_{0}^{(1)}+S_{0}-1$. Figure 1(b) shows that the CSD is exponential for large $\xi$ and Fig. 1(c) shows that for similarity coordinates the lines nearly collapse onto a single line and are therefore approximately self-similar. ${ }^{30}$ Eventually, the exponential CSD becomes a delta distribution.

Figure 2 shows the evolution of the supersaturation, $S$, and the parameter, $e^{\Omega}$, with time as they approach each other for $\lambda=1 / 3$. As indicated by Eq. (2.18), $S=e^{\Omega} \approx 1$ is required at equilibrium, where $d S / d \theta=0$. This justifies the expansion of $e^{\Omega}$ as a Taylor series for the asymptotic case, $t \rightarrow \infty$.

Figures 3 and 4 show the time evolution of the cluster number concentration and average cluster mass for various values of $\lambda$. The $\log -\log$ plots indicate power-law decrease, $C^{(0)} \sim \theta^{-b}$, and increase, $C^{\text {avg }} \sim \theta^{b}$, respectively, with time. 

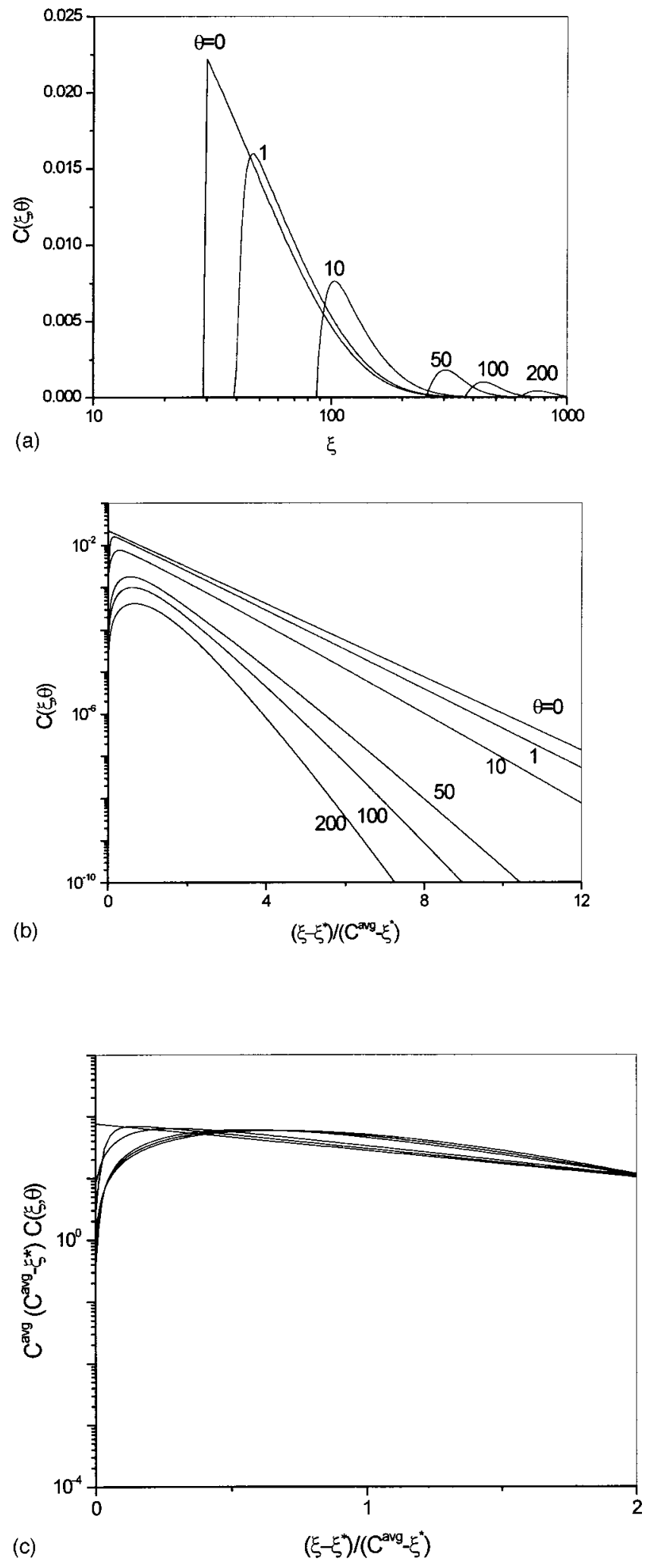

FIG. 1. Evolution of the dimensionless cluster size distribution for $\alpha=1$, $\omega=5, S_{0}=5, C_{0}^{(0)}=1, C_{0}^{\text {avg }}=75, \lambda=1 / 3$, plotted on (a) log-linear, (b) linear$\log$, and (c) similarity coordinates.

It is obvious that the increase in average particle mass is caused by the decrease in number of particles. If this were not the case, then the mass increase would come from a decrease in supersaturation, which is impossible if the system is near equilibrium $(S \approx 1)$. The powers approach the

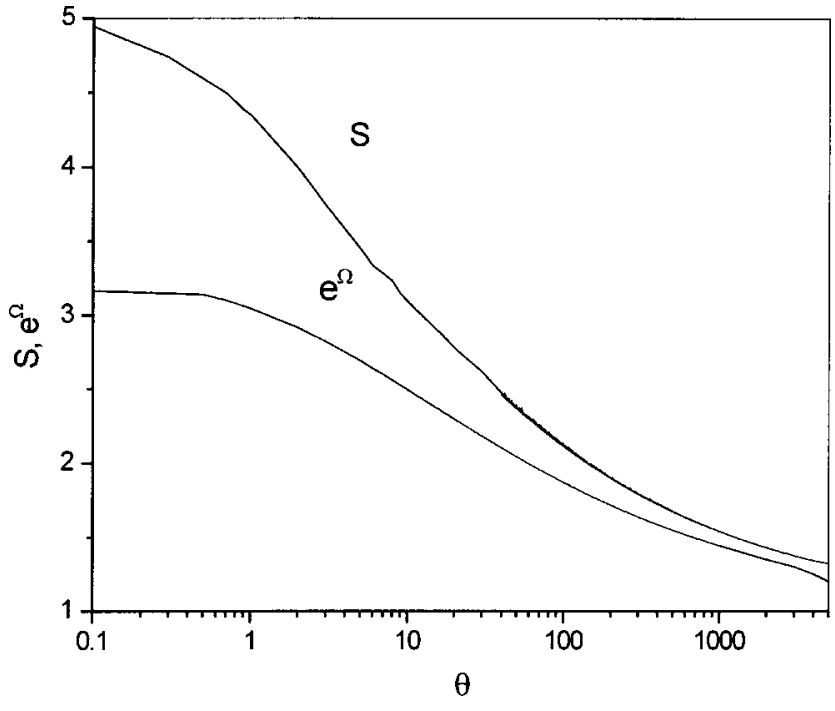

FIG. 2. Time dependence of $S(\theta)$ and $e^{\Omega}$ showing the decrease of the driving force, $S-e^{\Omega}$, for conditions of Fig. 1.

value in Table I, $b=1 /(4 / 3-\lambda)$, and can be derived as a long-time asymptotic solution as we will demonstrate shortly. The asymptotic slopes, identical to accepted values, ${ }^{21-23} b=1$ and $3 / 2$ for $\lambda=1 / 3$ and $2 / 3$, respectively, are independent of the initial conditions, as illustrated in Figs. 4(a)-4(c). The average radius of the spherical cluster is given by $4 r_{\mathrm{avg}}^{3} / 3 \pi=C^{\text {avg }}$, so that if we define $m$ by $r_{\mathrm{avg}}$ $\sim \theta^{1 / m}$, then $m=3 / b=(4-3 \lambda)$ as displayed in Table I. For $\lambda=1 / 3$ and $2 / 3$, the values are $m=3$ and 2 , and are approximately in the range for ripening and normal grain growth, ${ }^{23}$ from 2 to nearly 6. It is evident in Fig. 4 that for times smaller than the asymptotic limit, the slopes of $\log -\log$ plots of cluster size versus time are smaller, and $m$ is larger, reaching 6, as experiments show. ${ }^{23}$ For ripening experiments that deviate from ideal diffusion or surface control, a value of $\lambda$ could be selected to describe the data at a given time.

Figure 5 shows that the polydispersity, $C^{\text {pd }}$, for several values of $\lambda$, evolves to unity, corresponding to a delta distribution for a single large cluster remaining after infinite time.

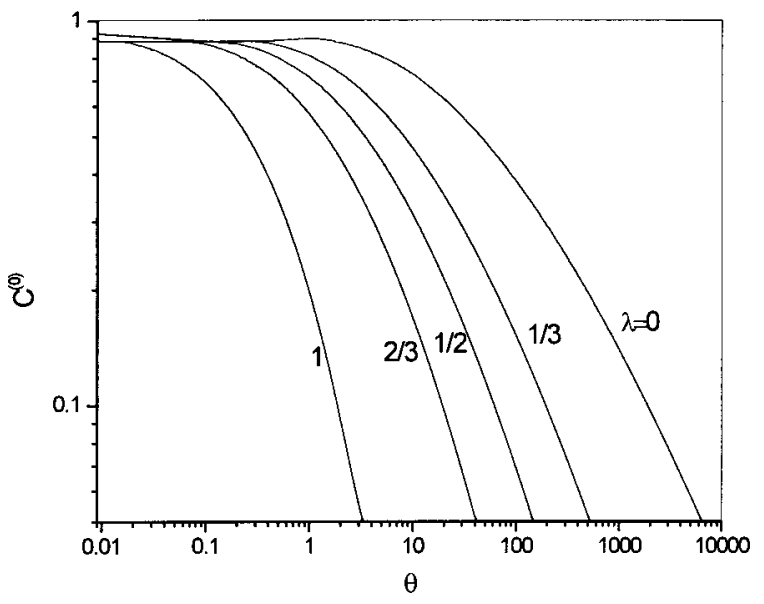

FIG. 3. Time evolution of the cluster number density, $C^{(0)}(\theta)$, showing asymptotic power-law decrease with time for various $\lambda$. The parameters used in the calculations are $\alpha=1, \omega=5, S_{0}=5, C_{0}^{\text {avg }}=75$ and $C_{0}^{(0)}=1$. 

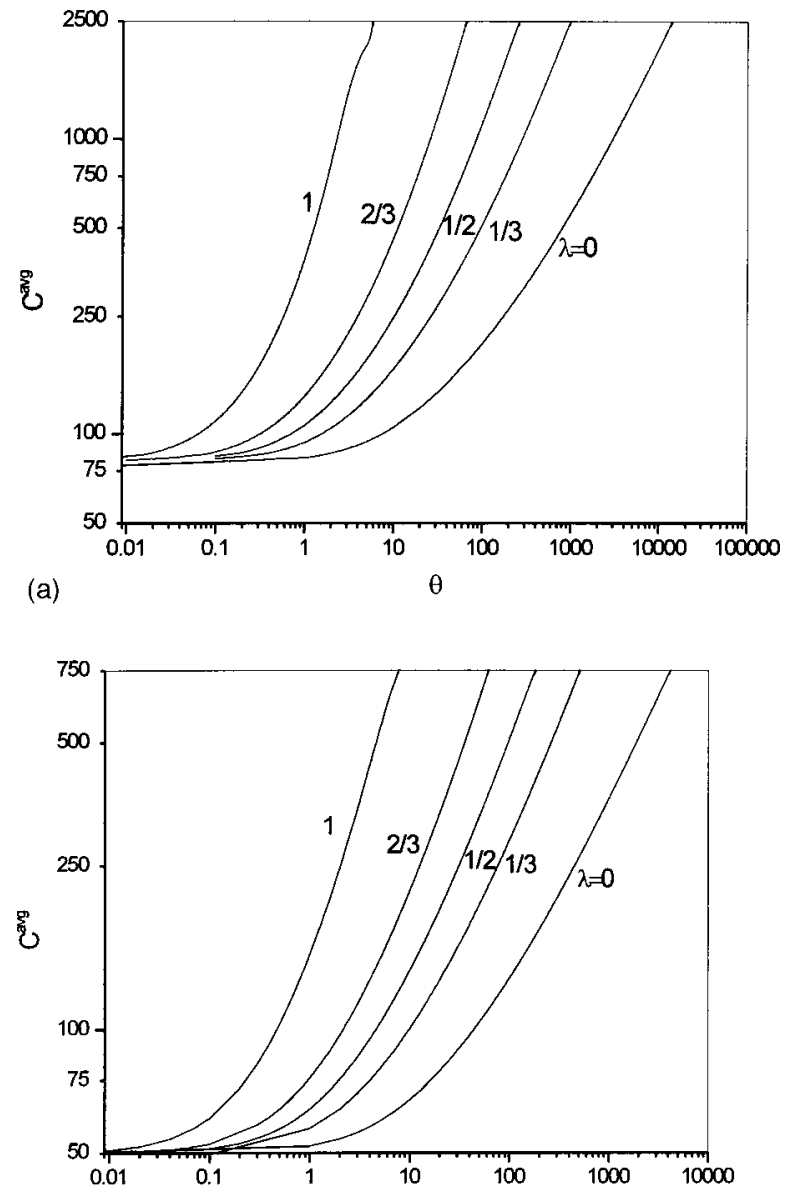

(b)

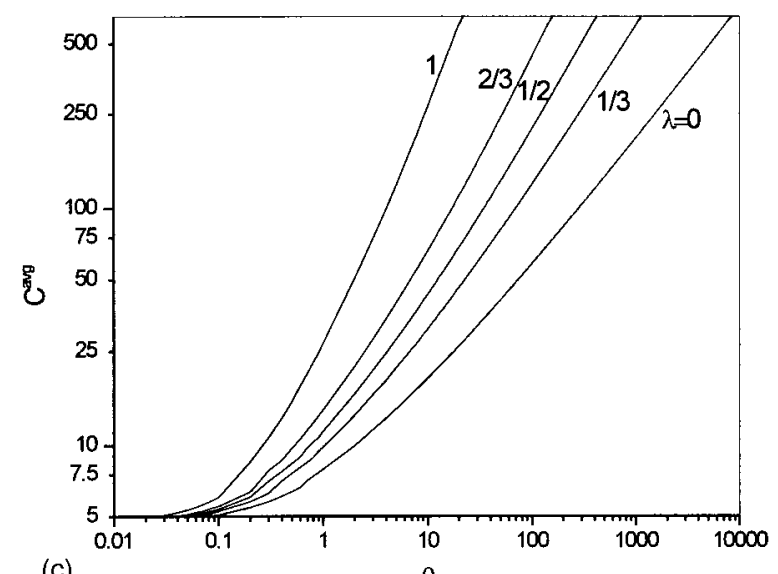

FIG. 4. Time evolution of the average cluster size, $C^{\text {avg }}(\theta)$, showing evolution to asymptotic power-law increase with time. The parameters used in the calculations are $\alpha=1, S_{0}=5$, and $C_{0}^{(0)}=1$ with (a) $\omega=5, C_{0}^{\text {avg }}=75$, (b) $\omega=5, C_{0}^{\mathrm{avg}}=50$, (c) $\omega=2, C_{0}^{\mathrm{avg}}=5$.

We also investigated the evolution of the CSDs for different initial distributions, by assuming various values of the gamma distribution parameter, $\alpha$, in Eq. (3.1) for $\lambda=1 / 3$. Changing $\alpha$ implies that the initial polydispersity is different in each case. Figure 6 indicates that the polydispersity, $C^{\mathrm{pd}}$, evolves to unity independently of the initial distribution.

\section{ASYMPTOTIC SOLUTION}

In this section we present an analytical asymptotic (longtime) scaling solution ${ }^{10,12}$ for the present approach to the
TABLE I. Power-law behavior of $C^{\mathrm{avg}} \sim \theta^{b}$ and $r_{\mathrm{avg}} \sim \theta^{1 / m}$.

\begin{tabular}{rcr}
\hline \hline$\lambda$ & $b=(4 / 3-\lambda)^{-1}$ & $m=3 / b$ \\
\hline 0 & $3 / 4$ & 4 \\
$1 / 3$ & 1 & 3 \\
$1 / 2$ & $6 / 5$ & $5 / 2$ \\
$2 / 3$ & $3 / 2$ & 2 \\
1 & 3 & 1 \\
\hline \hline
\end{tabular}

Ostwald ripening problem. We convert our PBE, Eq. (2.17), into a Fokker-Planck equation ${ }^{7}$ by expanding terms in $C(\xi$ $\pm 1, \theta)$ around large $\xi$ and keeping only first-order terms,

$$
\partial C(\xi, \theta) / \partial \theta=\partial\left[\xi^{\lambda}\left(S-e^{\Omega}\right) C(\xi, \theta)\right] / \partial \xi-J \delta\left(\xi-\xi^{*}\right) .
$$

The asymptotic solution requires that $S \rightarrow 1$ so that if we expand $e^{\Omega}$ we find

$$
e^{\Omega}-S \sim \omega / \xi^{1 / 3} \text {. }
$$

Because $\xi$ grows with time as the CSD shifts to larger particles, $\omega / \xi^{1 / 3}$ will eventually become small enough to justify keeping only one term in the expansion. The cluster-massbased Eq. (4.1) is similar to the cluster-radius-based conventional ripening equation, ${ }^{7}$ except that we have explicitly included the denucleation process.

The moment equations for Eq. (4.1) with Eq. (4.2) are found by multiplying by $\xi^{n}$ and integrating (the second term by parts) according to the moment definition, Eq. (2.20),

$$
d C^{(n)} / d \theta=-n \omega C^{(n+\lambda-4 / 3)}-J \xi^{* n}
$$

so that for $n=0$,

$$
d C^{(0)} / d \theta=-J
$$

and for $n=1$,

$$
d C^{(1)} / d \theta=-\omega C^{(\lambda-1 / 3)}-J \xi^{*} .
$$

Now we assume, following Binder ${ }^{12}$ and Marqusee and Ross, ${ }^{10}$ that for long time, $C(\xi, \theta)$ has a scaled solution in terms of a function $F$ to be determined,

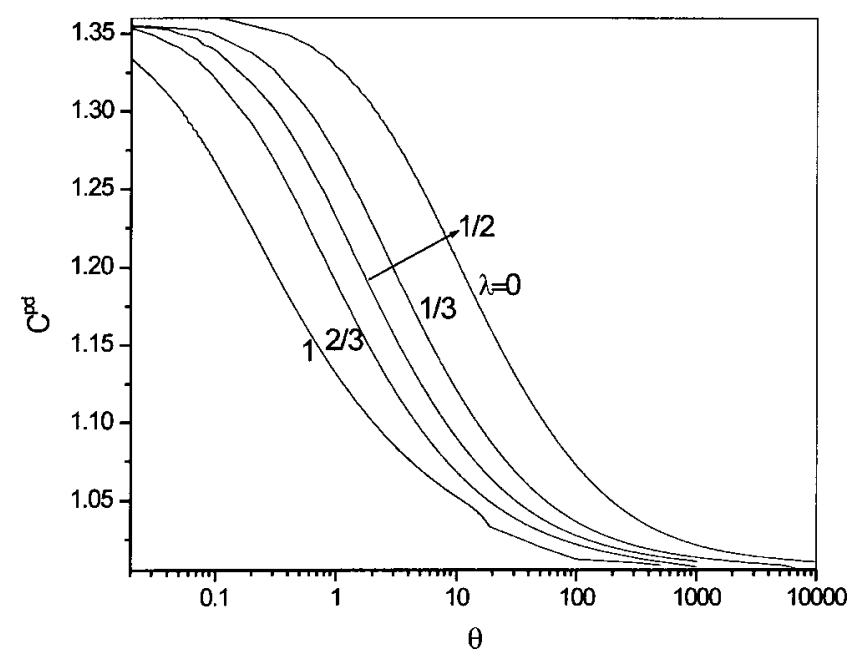

FIG. 5. Evolution of polydispersity, $C^{\mathrm{pd}}(\theta)$, with time. Other conditions are the same as in Fig. 3. 


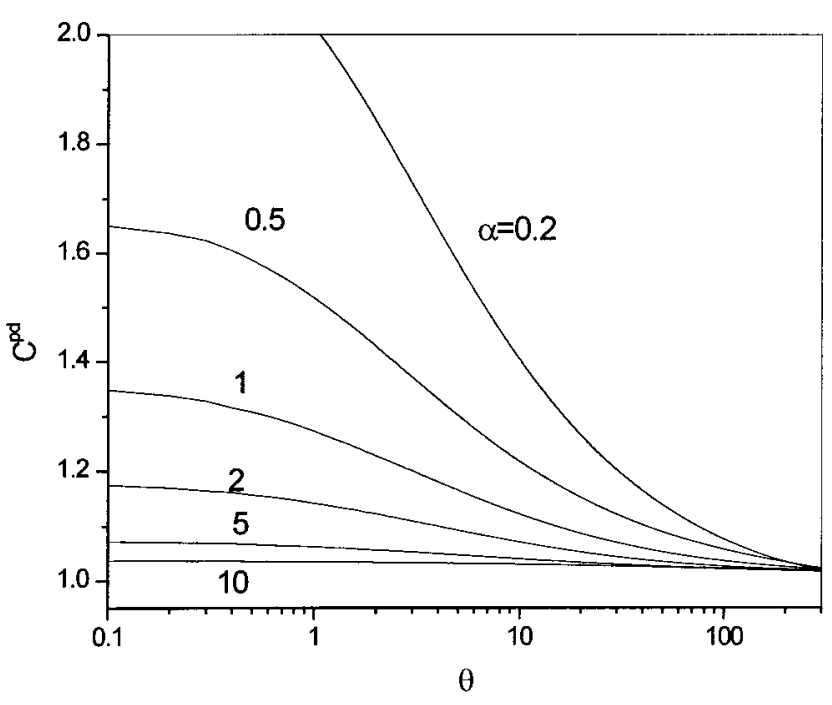

FIG. 6. Polydispersity, $C^{\mathrm{pd}}(\theta)$, as a function of time for various initial values of the gamma distribution parameter, $\alpha$. Other conditions are the same as in Fig. 1.

$$
C(\xi, \theta)=\theta^{-2 b} F\left(\left(\xi-\xi^{*}\right) \theta^{\chi}\right),
$$

which is indeed a general form for the exponential solution we found by our numerical analysis (Fig. 1),

$$
C(\xi, \theta)=\left[C^{(0)}(\theta) / \beta(\theta)\right] \exp \left[-\left(\xi-\xi^{*}\right) / \beta(\theta)\right] .
$$

Note that many ripening models have $\xi^{*}=0$, which in effect ignores the denucleation process. Because the growth and dissolution control the asymptotic behavior, such an approximation is admissible for a long-time solution, but becomes less accurate in the nonasymptotic range. Our numerical results show that

$$
C^{(0)}(\theta)=a_{0} \theta^{-b}
$$

and

$$
\beta(\theta)=C^{\mathrm{avg}}(\theta)-\xi^{*}=a_{1} \theta^{b},
$$

which implies that $C^{(1)}=C^{(0)} C^{\text {avg }}$ is constant with $\theta$ and $\xi^{*}(\theta) \sim \theta^{b}$. As time increases, the supersaturation $S$ decreases to unity, and by the mass balance, Eq. (3.1), the total cluster mass, $C^{(1)}$, becomes constant. This is referred to by Gratz $^{22}$ as "steady-state coarsening," although the system is changing with time. Therefore, the asymptotic solution is

$$
C(\xi, \theta)=\left[\theta^{-2 b} a_{0} / a_{1}\right] \exp \left[-\left(\xi-\xi^{*}\right) \theta^{-b} / a_{1}\right] .
$$

Thus we get the same relationship for $b$ and $\chi$ in Eq. (4.6) found by others; ${ }^{10,12}$ in our notation, $\chi=-b$. If $b$ is known, Eq. (4.10) provides the time and size dependence of the asymptotic CSD.

The moments of the exponential solution, Eq. (4.7), can be written for integer values of $n$,

$$
C^{(n)}(\theta)=C^{(0)}(\theta) \sum_{j=}^{n}\left(\begin{array}{c}
n \\
j
\end{array}\right) \xi^{* n-j} \beta^{j} j !,
$$

which yields the asymptotic time dependence, $C^{(n)}(\theta)$ $\sim \theta^{-b} \theta^{b n}$. With $d C^{(1)} / d \theta=0$ in Eq. (4.5), substituting the time dependence yields $\theta^{-b} \theta^{b(\lambda-1 / 3)} \sim \theta^{(-b-1)} \theta^{b}$, or

$$
b=1 /(4 / 3-\lambda) .
$$

To our knowledge, this is a new relationship between the asymptotic power, $b$, and the exponent, $\lambda$, of the rate coefficient. Equation (4.12) shows how the exponent on the mass dependence of the rate coefficients is related to the powerlaw behavior of the time-dependent growth during ripening. The new result [Eq. (4.12)] for average mass, $C^{\text {avg }}$ $\sim \theta^{1 /(4 / 3-\lambda)}$, is valid for well-known diffusion-controlled $(=1 / 3)$ and surface-controlled $(=2 / 3)$ processes, but may be useful for more complex kinetics as well. For example, by choosing the appropriate value of $\lambda$, one may model data with different values of $m .^{21}$

Because the CSD is sharply peaked, the result is the same if one considers a delta distribution in the asymptotic limit, $t \rightarrow \infty$, thus, if

$$
C(\xi, \theta)=C^{(0)}(\theta) \delta\left(\xi-\xi^{*}\right)
$$

then the moments are

$$
C^{(n)}(\theta)=C^{(0)}(\theta) \xi^{* n}
$$

and the solution proceeds as above. The asymptotic solution provides the basis for the assumed starting equation of other treatments. ${ }^{19-21}$

\section{CONCLUSION}

Mathematically modeling the Ostwald ripening problem is inherently difficult owing to the complex interactions of kinetics, thermodynamics, and distribution dynamics for the phase transition. Our approach utilizes a population balance equation that incorporates the kinetics of monomer addition and dissociation to describe how the cluster distribution evolves in time. The thermodynamic relationship for sizedependent solubility of clusters affects the driving force of supersaturation, and through detailed balancing, influences the mass dependence of the growth and dissolution rate coefficients. Small clusters are postulated to vanish spontaneously when they shrink to their critical nucleus size, thus reducing the number density of clusters during the evolution to a single large cluster. We thus differ from other models that assume clusters of all sizes are present, even those smaller than the critical nucleus size. By a numerical solution to the difference-differential population balance equation, Eq. (2.17), we find asymptotic power-law behavior, independent of initial conditions, for the decreasing cluster number density and for the increasing average cluster mass. According to an asymptotic solution based on the selfsimilar, exponential form of the long-time behavior, the power is simply related to the mass dependence of the rate coefficients as $b=(4 / 3-\lambda)^{-1}$.

A goal of the current work has been to examine carefully the detailed, fundamental features of Ostwald ripening. Consistent with prior distribution-kinetics models, ${ }^{6-9}$ the theory begins with the kinetics of the cluster mass distribution, which obeys an integrodifferential population balance equation. Moments of the PBE provide differential equations that show the evolution of total cluster mass, cluster number, and their ratio, average cluster mass. The PBE describes not only the reversible growth and dissolution rates, which depend on a power of the cluster mass, but also the denucleation process by which unstable clusters spontaneously dissolve and 
vanish. The ratio of interfacial to thermal energy gives the expression for the critical size when a cluster becomes unstable, and also provides the effect of particle-surface curvature on solubility. Although other theories of ripening do not adopt all these detailed features of kinetics and thermodynamics, they are mostly correct in the asymptotic limit when the growth process is rate-controlling.

${ }^{1}$ J. D. Ng, B. Lorber, J. Witz, A. T. Dietrich, D. Kern, and R. Giege, J. Cryst. Growth 168, 50 (1996).

${ }^{2}$ M. Kostoglou and A. G. Konstandopoulos, Aerosol. Sci. Technol. 32, 1399 (2001).

${ }^{3}$ D. Fan, L-Q. Chen, S. P. Chen, and P. W. Voorhees, Comput. Mater. Sci. 9, 329 (1998).

${ }^{4}$ O. Cavalerri, A. Hirstein, and K. Kern, Surf. Sci. 340, L960 (1995).

${ }^{5}$ A. C. Lasaga, Kinetic Theory in the Earth Sciences (Princeton University Press, Princeton, NJ, 1998).

${ }^{6}$ B. J. McCoy, J. Colloid Interface Sci. 228, 64 (2000).

${ }^{7}$ G. Madras and B. J. McCoy, J. Chem. Phys. 115, 6699 (2001).

${ }^{8}$ B. J. McCoy, J. Colloid Interface Sci. 240, 139 (2001).

${ }^{9}$ B. J. McCoy, Ind. Eng. Chem. Res. 40, 5147 (2001).

${ }^{10}$ J. A. Marqusee and J. Ross, J. Chem. Phys. 79, 373 (1983).
${ }^{11}$ A. W. Adamson and A. P. Gast, Physical Chemistry of Surfaces, 6th ed. (Wiley-Interscience, New York, 1997).

${ }^{12}$ K. Binder, Phys. Rev. B 15, 4425 (1977).

${ }^{13}$ G. Feher and Z. Kam, Methods Enzymol. 114, 77 (1985).

${ }^{14}$ B. J. McCoy and G. Madras, J. Colloid Interface Sci. 201, 200 (1998).

${ }^{15}$ M. Zinke-Allmang, L. C. Feldman, and M. H. Grabow, Surf. Sci. Rep. 16, 377 (1992).

${ }^{16}$ M. Zinke Allmang, Thin Solid Films 346, 1 (1993).

${ }^{17}$ P. Neogi and G. Narsimhan, Chem. Eng. Sci. 56, 4225 (2001).

${ }^{18}$ I. M. Lifshitz and V. V. Slyozov, J. Phys. Chem. Solids 19, 35 (1961).

${ }^{19}$ C. Wagner, Z. Elektrochem. 65, 581 (1961).

${ }^{20}$ A. Bhakta and E. Ruckenstein, J. Chem. Phys. 103, 7120 (1995).

${ }^{21}$ H. Gratz, Scr. Mater. 34, 1209 (1996).

${ }^{22}$ H. Gratz, Scr. Mater. 37, 9 (1997).

${ }^{23}$ H. Gratz, J. Mater. Sci. Lett. 18, 1637 (1999).

${ }^{24}$ H. Xia and M. Zinke-Allmang, Physica A 261, 176 (1998).

${ }^{25}$ F. Reif, Fundamentals of Statistical and Thermal Physics (McGraw-Hill, New York, 1965).

${ }^{26}$ S. R. de Groot and P. Mazur, Non-Equilibrium Thermodynamics (NorthHolland, Amsterdam, 1962)

${ }^{27}$ M. von Smoluchowski, Z. Phys. 17, 585 (1916).

${ }^{28}$ D. F. Calef and J. M. Deutch, Annu. Rev. Phys. Chem. 34, 493 (1983).

${ }^{29}$ T. Olson and P. Hamill, J. Chem. Phys. 104, 210 (1996)

${ }^{30}$ G. R. Carlow and M. Zinke-Allmang, Phys. Rev. Lett. 78, 4601 (1997). 